Journal for ImmunoTherapy of Cancer

\title{
Peptide vaccine targeting mutated GNAS: a potential novel treatment for pseudomyxoma peritonei
}

\author{
Kjersti Flatmark (D) , ${ }^{1,2,3}$ Annette Torgunrud (D) ,,3 Karianne G Fleten (D) ,,3 \\ Ben Davidson (D) , ${ }^{2,4}$ Hedvig V Juul, ${ }^{5}$ Nadia Mensali (D) , \\ Christin Lund-Andersen (D) , 2,3 Else Marit Inderberg (iD ${ }^{5}$
}

To cite: Flatmark $\mathrm{K}$, Torgunrud A, Fleten KG, et al. Peptide vaccine targeting mutated GNAS: a potential novel treatment for pseudomyxoma peritonei. Journal for ImmunoTherapy of Cancer 2021;9:e003109. doi:10.1136/ jitc-2021-003109

\section{- Additional supplemental} material is published online only. To view, please visit the journal online (http://dx.doi.org/10. 1136/jitc-2021-003109).

Accepted 27 September 2021

Check for updates

(c) Author(s) (or their employer(s)) 2021. Re-use permitted under CC BY-NC. No commercial re-use. See rights and permissions. Published by BMJ.

\section{Department of}

Gastroenterological Surgery, Oslo University Hospital, Oslo, Norway

${ }^{2}$ Institute of Clinical Medicine, University of Oslo, Oslo, Norway ${ }^{3}$ Department of Tumor Biology, Oslo University Hospital, Oslo, Norway

${ }^{4}$ Department of Pathology, 0slo University Hospital, Oslo, Norway ${ }^{5}$ Department of Cellular Therapy, Department of Oncology, Oslo University Hospital, Oslo, Norway

Correspondence to

Dr Kjersti Flatmark;

kjersti.flatmark@rr-research.no

\section{ABSTRACT}

Background Pseudomyxoma peritonei (PMP) is a rare, slow-growing abdominal cancer with no efficacious treatment options in non-resectable and recurrent cases. Otherwise, rare activating mutations in the GNAS oncogene are remarkably frequent in PMP and the mutated gene product, guanine nucleotide-binding protein $\alpha$ subunit (Gs $\alpha$ ), is a potential tumor neoantigen, presenting an opportunity for targeting by a therapeutic cancer vaccine. Methods Tumor and blood samples were collected from 25 patients undergoing surgery for PMP (NCT02073500). GNAS mutation analysis was performed by next-generation targeted sequencing or digital droplet PCR. Responses to stimulation with Gso mutated (point mutations R201H and R201C) 30 mer peptides were analyzed in peripheral blood T cells derived from patients with PMP and healthy donors. Fresh PMP tumor samples were analyzed by mass cytometry using a panel of 35 extracellular markers, and cellular subpopulations were clustered and visualized using the visual stochastic network embedding analysis tool.

Results GNAS mutations were detected in 22/25 tumor samples (88\%; R201H and R201C mutations detected in 16 and 6 cases, respectively). Strong T cell proliferation against Gs $\alpha$ mutated peptides was observed in 18/24 patients with PMP. Mass cytometry analysis of tumor revealed infiltration of $\mathrm{CD} 3+\mathrm{T}$ cells in most samples, with variable CD4+:CD8 + ratios. A large proportion of T cells expressed immune checkpoint molecules, in particular programmed death receptor-1 and T cell immunoreceptor with Ig and ITIM, indicating that these T cells were antigen experienced.

Conclusion These findings point to the existence of a preexisting immunity in patients with PMP towards mutated Gs $\alpha$, which has been insufficient to control tumor growth, possibly because of inhibition of antitumor T cells by upregulation of immune checkpoint molecules. The results form a rationale for exploring peptide vaccination with Gso peptides in combination with immune checkpoint inhibiton as a possible curative treatment for PMP and other GNAS mutated cancers.

\section{BACKGROUND}

Pseudomyxoma peritonei (PMP) is a rare, slow-growing abdominal cancer that commonly originates in ruptured appendiceal mucinous neoplasms, seeding tumor cells, and mucin into the peritoneal cavity. The disease is characterized by slow, progressive accumulation of mucinous tumor tissue in the peritoneal cavity, ultimately leading to abdominal compression. Standard-of-care treatment involves cytoreductive surgery (CRS) to remove all visible tumor tissues, followed by hyperthermic intraperitoneal chemotherapy (HIPEC) to eliminate microscopic residual disease. ${ }^{1-3}$ This treatment is curative in approximately $50 \%$ of the patients, but for patients who cannot be cured by CRS-HIPEC, no efficacious treatment options exist. Responses to systemic chemotherapy are generally poor, and biological agents, such as angiogenesis inhibitors, have been suggested as alternative therapeutic approaches, but so far with little success. In the setting of non-resectable and recurrent disease, PMP is a debilitating and ultimately fatal condition, leaving patients to experience progressively poor quality of life caused by an increasing intraperitoneal tumor burden.

A remarkably high frequency $(60 \%-100 \%)$ of otherwise infrequently occurring mutations in the GNAS oncogene points to GNAS as a potential therapeutic target in PMP. ${ }^{4}$ GNAS encodes the guanine nucleotidebinding protein $\alpha$ subunit $(G s \alpha)$, which functions as a molecular switch to control cell growth, survival, and motility. The mutations are located in codon 201 (R201C and $\mathrm{R} 201 \mathrm{H})$ and result in constitutive activation of downstream signaling through the protein kinase A pathway. This activation may explain the massive production of mucin and suggests that mutated GNAS is a major oncogenic driver in PMP. Interestingly, the same GNAS mutations are found in subgroups of several other cancer entities (overall estimated frequency of $4.4 \%$ ), making this the 
most frequently mutated $G$ protein in cancer, further supporting the potential value of a therapeutic intervention directed at this target. ${ }^{56}$

The intracellular location of Gs $\alpha$ combined with the single-codon mutation makes this a difficult target for small-molecule inhibitors and antibodies, which might explain why no targeted therapies exist. A vaccine-based treatment targeting mutated GNAS could therefore be a valuable addition to the cancer treatment toolbox with a much broader scope than PMP alone.

Mutations in cancer driver genes may act as neoantigens and are particularly interesting targets for development of therapeutic cancer vaccines, and patients with slow-growing tumors represent an ideal clinical setting. Therefore, a vaccination approach targeting mutated GNAS could represent a novel therapeutic opportunity in the hope of providing a cure for PMP. To explore mutated GNAS as a target for a cancer vaccine approach, we first questioned if long, synthetic Gs $\alpha$ peptides carrying the relevant mutations are immunogenic by analyzing responses in peripheral blood $\mathrm{T}$ cells derived from patients with PMP. We further investigated immune cell infiltration in the PMP tumor microenvironment in tumor samples collected at the time of surgery from the same patients and performed immunoprofiling by mass cytometry on immune cells isolated from tumor specimens.

\section{METHODS \\ Clinical samples}

Patients with suspected PMP were included in the study between April 2018 and May 2020 when admitted for CRS-HIPEC at the Norwegian Radium Hospital, Oslo University Hospital Comprehensive Cancer Center. Tumor and peripheral blood samples were collected at the time of surgery. Tumor distribution on the peritoneal surface was classified by the surgeon according to the Peritoneal Cancer Index (PCI), giving a score between 0 and 39. ${ }^{7}$ Residual tumor after CRS was classified using the completeness of cytoreduction (CC) score (CC-0, no residual tumor; CC-1, residual tumor $<0.25 \mathrm{~cm}$; CC-2, tumor between $0.25 \mathrm{~cm}$ and $2.5 \mathrm{~cm}$ and CC-3, tumor $>2.5 \mathrm{~cm}) .{ }^{7}$ Complete cytoreduction was defined as CC-0 and CC-1. All PMP cases were evaluated by an expert pathologist and classified according to the Peritoneal Surface Oncology Group International classification. ${ }^{8}$ Peripheral blood was collected from anonymous healthy donors for testing of immune responses on informed consent (project ID \#2019/121).

\section{Analysis of tumor GNAS and KRAS mutations}

Fresh tumor samples were collected at the time of surgery from 22/25 cases. Samples were immediately snap frozen and stored at $-80^{\circ} \mathrm{C}$ until further processing. The tumor content was assessed in H\&E-stained frozen sections. Regardless of tumor content, available samples were homogenized and disrupted using TissueLyzer LT
(Qiagen, Hilden, Germany). DNA was extracted using the AllPrep DNA/RNA/miRNA Universal Kit, automated with the use of QIAcube (Qiagen). For 10 PMP cases, no fresh tumor tissue was collected at CRS-HIPEC $(n=3)$ or no mutation was detected in fresh-frozen samples with no or very low tumor cell content $(n=7)$. In these cases, DNA was additionally extracted from the formalin fixed, paraffin-embedded routine pathology samples of the peritoneal disease or the primary appendiceal tumor after microdissection, using the QIAcube and AllPrep DNA/ RNA formalin-fixed paraffin-embedded (FFPE) Kit. DNA purity was measured using NanoDrop (Thermo Fisher Scientific, Waltham, Massachusetts, USA); median absorbance ratio 260/280 was 1.83 (min-max 1.51-2.55), and concentrations were determined with the Qubit fluorometer (Thermo Fisher Scientific). Targeted next-generation sequencing was performed with the Ion GeneStudio S5 system and the Oncomine Comprehensive Assay V.3 (Thermo Fisher Scientific), covering single-nucleotide variants and indels from 161 unique genes. The median coverage of called variants was 4929 , enabling detection of variants down to $1 \%$ allele frequency. Variants were called, annotated, and filtered with Ion Reporter Software V.5.10 (Thermo Fisher Scientific) and manually reassessed using Integrative Genomics Viewer. In DNA from the formalin-fixed samples, the presence of $G N A S$ and KRAS mutations was assessed using the digital droplet PCR (ddPCR) system from BioRad (BioRad, Hercules, California, USA). The ddPCR Mutation Assay (GNAS R201H (assayID: dHsaMDV2516792) and GNAS R201C (assayID: dHsaMDV2510562)) were used for detection of R201H and R201C mutations, respectively. For detection of KRAS G12D, G12V, and G13D mutations, the KRAS G12D (assayID: dHsaMDV2510596), KRAS G12V (assayID: dHsaMDV2510592), and KRAS G13D (assayID: dHsaMDV2510598) assays were used. Premix preparation, droplet generation, and thermal cycling were performed according to the manufacturer's instructions. The fluorescence intensity in droplets was detected by a QX200 Droplet Reader (BioRad). For both assays, a 'no template control' and a positive control were included for quality control. QuantaSoft V.1.7.4 analysis software and QX Manager Software (BioRad) was used for data acquisition and analysis. Only tests providing $>13.000$ droplets were considered valid.

\section{T cell proliferation assays}

Peripheral blood mononuclear cells (PBMCs) from patients with PMP and healthy donors were isolated and frozen and later thawed for testing as previously described. ${ }^{9}$ PBMCs were stimulated once in vitro with $10 \mu \mathrm{M}$ Gs $\alpha$ mutated peptides at $2 \times 10^{6}$ cells $/ \mathrm{mL}$ in X-Vivo 15 medium (Lonza, Basel, Switzerland), adding 20U/ mL IL-2 (Clinigen, Burton on Trent, UK) and $5 \mathrm{ng} / \mathrm{mL}$ IL-7 (Bio-Techne, Minneapolis, Minnesota, USA) on day 3. The Gs $\alpha$ peptides (aa 186-215) contained point mutations R201H and R201C (ProImmune, Oxford, UK) and were of indicated lengths (30 amino acids). After 
12-14days, $\mathrm{T}$ cells were tested in proliferation assays; prestimulated $\mathrm{T}$ cells were seeded at $5 \times 10^{4}$ cells per well in 96-well round-bottomed plates. The same number of irradiated (30 Gy), autologous PBMCs was added for use as antigen-presenting cells and Gs $\alpha$ mutated peptides as well as the wild-type (WT) peptide sequence were added at $10 \mu \mathrm{M}$. Staphylococcal superantigen SEC3 $(0.1 \mu \mathrm{g} /$ $\mathrm{mL}$ ) was added as a positive control. Proliferation was measured on day 3 after labeling with $3.7 \times 10^{4} \mathrm{~Bq}{ }^{3} \mathrm{H}$-Thymidine (Montebello Diagnostics AS, Oslo, Norway) overnight before harvesting. All conditions were tested in triplicate. The Stimulatory Index (SI) was defined as proliferation with peptide divided by proliferation without peptide and SD were calculated. SI $\geq 2$ was considered as a positive response.

\section{Interferon gamma (IFN- $\gamma$ ) enzyme-linked immunospot (ELISPOT) assays}

If cell numbers were sufficient with either freshly peptide prestimulated or thawed prestimulated $\mathrm{T}$ cells, IFN-y ELISPOT assays (CTL Europe GmbH, Bonn, Germany) were performed. $\mathrm{T}$ cells and autologous PBMCs were plated onto plates precoated with antihuman IFN-y at $1: 1$ ratio $\left(0.5-1.0 \times 10^{5}\right.$ cells) and $10 \mu \mathrm{M}$ of 30 mer Gs $\alpha$ peptides (WT, R201C, and R201H) were added. Wells with no stimulus served as a negative control, while cells stimulated with $0.1 \mu \mathrm{g} / \mathrm{mL}$ SEC3 served as a positive control. Plates were incubated for 24 hours prior to addition of detection reagents and substrate following the manufacturer's instructions. The plates were imaged and the spot counts determined using an automated ELISPOT analyzer ImmunoSpot S6ULTIMATE (Cellular Technology, Shaker Heights, USA). Peptide-specific spot counts were determined by subtracting the mean spot number of no peptide-stimulated coincubated $T$ cells and PBMC control wells for each patient from the number of spots in the Gs $\alpha$ peptide (WT, R201C, and R201H) stimulated wells. As there were insufficient cell numbers to perform titrations (except for one patient), saturated well counts for SEC3-positive controls where spots were too numerous to count (TNT) were set to $>1000$ spots or TNTC. Raw data included images for each well for visual quality checking (online supplemental figure 1).

\section{Mass cytometry (cytometry by time of flight (CyTOF)) analysis of immune cells from PMP tissues}

Fresh tumor tissue was disaggregated with Collagenase II (Sigma-Aldrich, St Louis, Michigan, USA) and DNase I (Sigma-Aldrich) after cutting tumor into small pieces. The single-cell suspension was then washed and erythrocytes were lysed by adding ACK Lysis Buffer (Thermo Fisher Scientific) before a second wash. The single cells were frozen in fetal bovine serum (Gibco, Thermo Fisher Scientific) containing 12\% dimethyl sulfoxide (SigmaAldrich) and stored in liquid nitrogen. Briefly, single cells from biopsies were thawed, washed, and resuspended in MaxPar cell staining buffer (Fluidigm, San Francisco, California, USA), before staining with Cell-ID cisplatin
(Fludigm) for $5 \mathrm{~min}$, then washed and stained with extracellular antibodies for $30 \mathrm{~min}$. The samples were then fixed with $1.6 \%$ paraformaldehyde and permeabilized in $99 \%$ methanol (Sigma-Aldrich). Samples were stored at $-80^{\circ} \mathrm{C}$ in methanol for up to 4 weeks. After removing methanol, all samples were incubated with iridium cell tracker (Fluidigm) for $20 \mathrm{~min}$, washed and resuspended in water with $10 \%$ of calibration beads (Fluidigm). Samples were filtered immediately before sample acquisition on a CyTOF 2 (Fluidigm) instrument at the OUS Flow Cytometry core facility. Analysis was performed using Cytobank Cellmass (cytobank.org). A panel of 35 extracellular markers was used (see online supplemental table 1). Typical gating strategy was applied as follows: (1) EQ-140 versus Ir-191 to exclude calibration beads, (2) Ir-191 versus Ir-193 to gate singlets, (3) Ir-191 versus event length to gate intact singlet, and (4) cisplatin versus CD45-89Y to gate live lymphocytes. All further analyses were carried out on this population. Cellular subpopulations were clustered and visualized using the (visual stochastic network embedding (viSNE) analysis tool (based on the t-distributed stochastic neighbor embedding clustering algorithm). The clustering was based on the expression of CD45RA, CD19, CD11b, CD4, CD20, CD21, IgD, CD14, CD8a, CD3, HLA-DR, CD56, CD16, CCR6, CD25, PD-L1, PD-L2, lymphocyte-activation gene 3 (LAG-3), TIM-3, T cell immunoreceptor with Ig and ITIM (TIGIT), programmed death receptor-1 (PD-1), CCR7, CD28, CTLA-4, ICOS, CXCR3, CXCR5, CXCR4, CD161, CD127, NKG2D, CD38, and CD33 and ran with the following parameters: 1000 iterations, 30 perplexities and 0.5 theta.

Flow cytometry analysis of immune cells from PMP tissues

Single cells from biopsies were thawed, washed and resuspended in staining buffer (phosphate buffered saline with $2 \%$ fetal calf serum) at $0.2-0.5 \times 10^{6}$ per tube. Ten microlitres of aggregated gamma-globulin (Fc receptor block) was added and left for $15 \mathrm{~min}$ at room temperature (RT). Antibodies were added and the samples were incubated at RT for another 20 min in the dark. Antibodies used were CD3 FITC (OKT3; eBioscience, San Diego, California, USA), CD4 BV421 (OKT4; BioLegend, San Diego, California, USA), CD8 BV605 (RPA-T8, BioLegend), CD39 APC (eBioA1, eBiosciences), CD69 PE-Cy7 (FN50, BioLegend), TIGIT PE-Cy7 (MBSA43, eBioscience), and PD-1 PE-Cy7 (eBioJ105, eBioscience)

Cells were then washed in staining buffer prior to direct acquisition on a FACS Canto II (BD Biosciences). Data were analyzed using FlowJo software (Treestar, Ashland, USA). The gating strategy was the following: the lymphocytes were selected from the scatter gate in forward scatter (FSC) versus side scatter (SSC) dot plot, then $\mathrm{CD} 4$ and $\mathrm{CD} 8$ gates were set in a $\mathrm{CD} 4$ versus CD8 dot plot before gating out CD39 and CD69 single or double positive populations for CD8 + or CD4+ cells in a CD39 versus CD69 dot plot. Cells were also stained for PD-1 and TIGIT to confirm that the percentages of 
positive cells were similar to what was obtained in mass cytometry.

\section{Statistical analysis}

Paired, two-tailed t-tests were used to compare $\mathrm{T}$ cell proliferation against the WT Gs $\alpha$ peptide with each of the other conditions. Paired, two-tailed t-tests were also used to compare T cell IFN- $\gamma$ production against the WT Gs $\alpha$ peptide with each of the other conditions for each patient. All statistical analyses were performed using GraphPad Prism V.8 software (GraphPad Software, San Diego, California, USA).

\section{RESULTS}

\section{Patients}

Twenty-five patients with PMP were included in the study, 16 women and 9 men, with a median age of 55 years (min-max 32-74) (table 1). Of these, 23 had appendiceal primary tumors (classified as low-grade mucinous neoplasm, $\mathrm{n}=17$; high-grade mucinous neoplasm, $\mathrm{n}=3$; mucinous adenocarcinoma, $n=2$; and not classified, $n=1$ ), while two patients had primary mucinous adenocarcinomas of the ovary. On histological examination of the peritoneal disease, 22 cases were classified as low-grade PMP (of these, 9 contained acellular mucin only), 2 as high-grade, and 1 as high-grade with signet-ring cells. The median PCI was 25 (min-max, 2-39). For 20 patients, complete cytoreduction was achieved (CC0/1), and these

Table 1 Key clinicopathological parameters, mutational status and T cell proliferation score for patients with PMP

\begin{tabular}{|c|c|c|c|c|c|c|c|c|c|c|c|c|}
\hline \multirow[b]{2}{*}{$\begin{array}{l}\text { Case } \\
\text { number }\end{array}$} & \multirow[b]{2}{*}{ Sex } & \multirow[b]{2}{*}{ Age } & \multirow[b]{2}{*}{$\begin{array}{l}\text { Primary } \\
\text { tumor }\end{array}$} & \multirow[b]{2}{*}{$\mathrm{PCI}$} & \multirow[b]{2}{*}{ HIPEC } & \multicolumn{2}{|c|}{$\begin{array}{l}\text { Histological } \\
\text { classification }\end{array}$} & \multirow[b]{2}{*}{$\begin{array}{l}\text { GNAS } \\
\text { mutation }\end{array}$} & \multirow[b]{2}{*}{$\begin{array}{l}\text { KRAS } \\
\text { mutation }\end{array}$} & \multicolumn{3}{|c|}{$\begin{array}{l}\text { Proliferation score } \\
\text { according to peptide }\end{array}$} \\
\hline & & & & & & $\begin{array}{l}\text { Primary } \\
\text { tumor }\end{array}$ & $\begin{array}{l}\text { Peritoneal } \\
\text { disease }\end{array}$ & & & $\begin{array}{l}\text { Wild } \\
\text { type }\end{array}$ & R201C & R201H \\
\hline 434 & M & 55 & Appendix & 32 & Yes & LAMN & LG & $\mathrm{R} 201 \mathrm{H}$ & G12D & \multicolumn{3}{|c|}{ Not performed* } \\
\hline 440 & $\mathrm{~F}$ & 47 & Appendix & 3 & Yes & LAMN & LG† & $\mathrm{R} 201 \mathrm{H}$ & G12D & 1.5 & 4.4 & 5.7 \\
\hline 445 & $\mathrm{~F}$ & 70 & Appendix & 7 & Yes & LAMN & LG & R201C & G13D & 4.4 & 20.9 & 2.7 \\
\hline 519 & $\mathrm{~F}$ & 60 & Appendix & 2 & Yes & LAMN & LG† & R201C & NMD & 0.9 & 1.3 & 1.2 \\
\hline 522 & M & 38 & Appendix & 11 & Yes & HAMN & LG & $\mathrm{R} 201 \mathrm{H}$ & G12D & 1.0 & 0.9 & 1.6 \\
\hline 523 & $\mathrm{~F}$ & 61 & Appendix & 39 & No & MA & PMCA-S & NMD & NMD & 0.6 & 2.9 & 1.6 \\
\hline 530 & $\mathrm{~F}$ & 56 & Appendix & 38 & No & HAMN & LG & R201C & G12V & 9.4 & 16.8 & 16.1 \\
\hline 547 & M & 48 & Appendix & 39 & Yes & LAMN & LG† & $\mathrm{R} 201 \mathrm{H}$ & G12V & 1.1 & 1.1 & 0.6 \\
\hline 549 & M & 32 & Appendix & 13 & Yes & LAMN & LG† & $\mathrm{R} 201 \mathrm{H}$ & G12D & 0.9 & 4.6 & 0.5 \\
\hline 558 & $\mathrm{~F}$ & 37 & Ovary & 6 & Yes & MA & LG & $\mathrm{R} 201 \mathrm{H}$ & G12D & 1.4 & 1.0 & 0.2 \\
\hline 559 & M & 54 & Appendix & 24 & Yes & LAMN & LG† & NMD & NMD & 5.4 & 35.4 & 0.3 \\
\hline 563 & $\mathrm{~F}$ & 50 & Appendix & 23 & Yes & MA & $H G$ & $\mathrm{R} 201 \mathrm{H}$ & G12D & 6.2 & 15.3 & 10.4 \\
\hline 570 & $\mathrm{~F}$ & 70 & Appendix & 2 & Yes & HAMN & LG† & $\mathrm{R} 201 \mathrm{H}$ & $\begin{array}{l}\text { G13D/ } \\
\text { G12D }\end{array}$ & 1.1 & 16.8 & 13.6 \\
\hline 572 & M & 46 & Appendix & 30 & Yes & LAMN & $\mathrm{LG} \dagger$ & R201C & NMD & 2.4 & 10.2 & 28.3 \\
\hline 575 & $\mathrm{M}$ & 72 & Appendix & 35 & No & LAMN & $\mathrm{LG}$ & R201C & G12V & 0.9 & 1.1 & 1.2 \\
\hline
\end{tabular}

*Blood samples not available, CyTOF analysis only.

†Only acellular mucin detected at microscopy of resected peritoneal tumor.

†Primary tumor suspected to be appendiceal on radiology, no surgical specimen available.

CyTOF, cytometry by time of flight; F, female; HAMN, high-grade mucinous neoplasm; HG, high-grade; LAMN, low-grade mucinous neoplasm; LG, low-grade; M, male; MA, mucinous adenocarcinoma; NMD, no mutation detected; PCI, Peritoneal Cancer Index; PMCA-S, peritoneal mucinous carcinomatosis with signet-ring cells; PMP, pseudomyxoma peritonei. 
patients received mitomycin C-based HIPEC, while in 5 cases, palliative procedures were performed and no HIPEC was given.

\section{Tumor mutation analysis}

GNAS mutations were detected in samples from 22/25 patients $(88 \%)$, while in three cases, no mutation was found. The R201H and R201C mutations were detected in 16 and 6 cases, respectively (table 1 ). KRAS mutations, which are also commonly present in PMP, were detected in $21 / 25$ samples $(84 \%)$, with no mutations in four cases (G12D, n=12; G13D, n=3; G12V, n=5; both G12D and
G13D, n=1). In most cases, the two genes were comutated (in 20 of the 25 analyzed cases); in two cases, no mutations were detected in either gene, and in three cases, only one gene was mutated ( GNAS only, $\mathrm{n}=2$; KRAS only, $\mathrm{n}=1)$.

\section{Immune stimulation of T cells from peripheral blood samples}

PBMC samples were available from 24 of the 25 patients and from 10 healthy donors. When stimulated with the mutated peptides, proliferative $\mathrm{T}$ cell responses against one or both peptides were observed in 18/24 PMP samples (table 1 and figure 1A). A trend towards

A

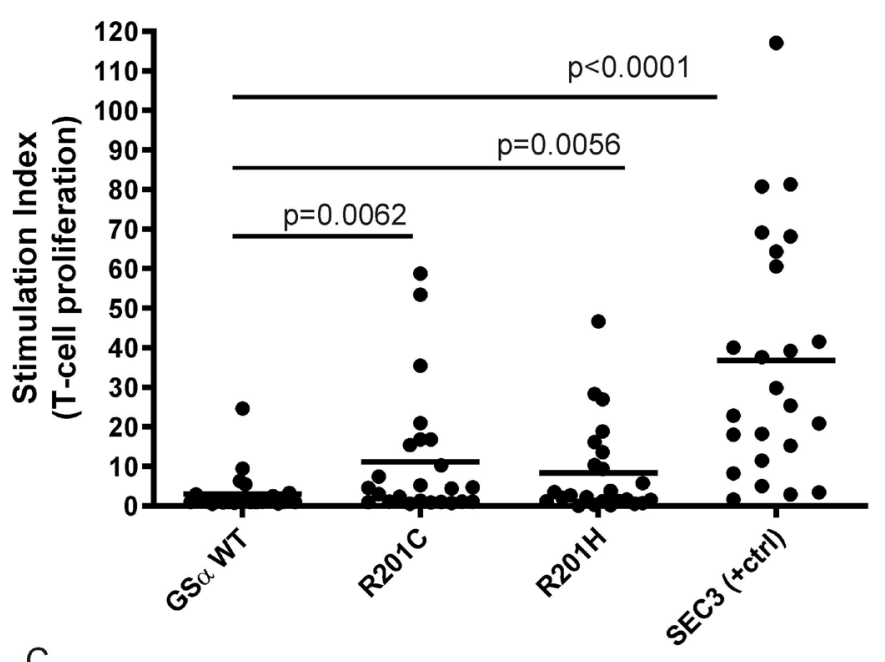

B

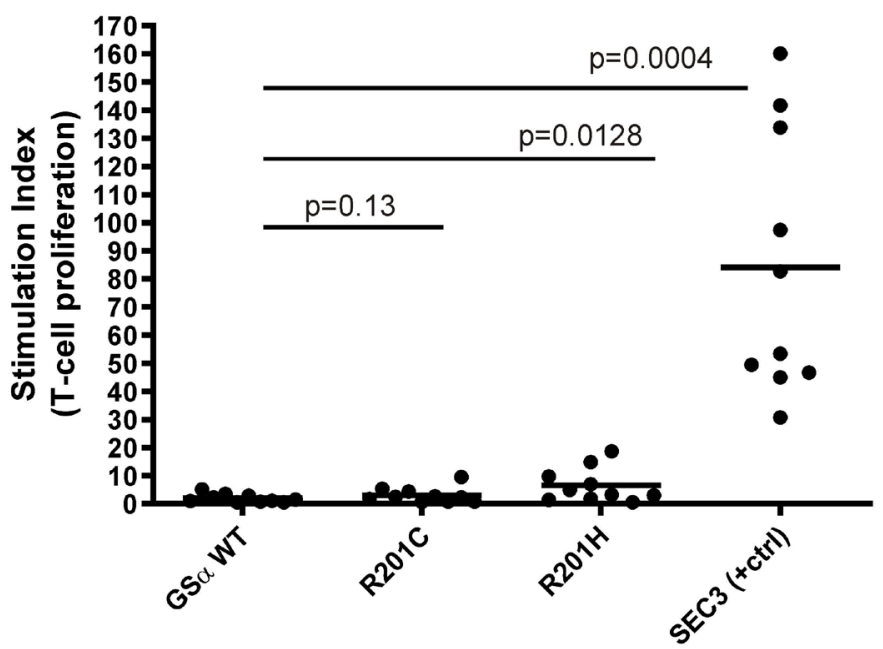

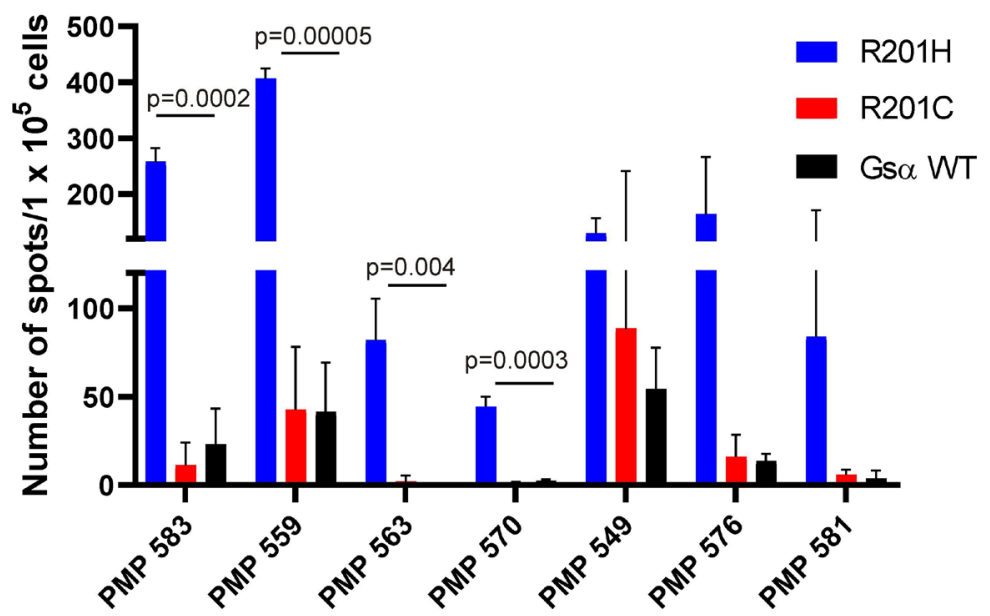

Figure 1 Mutated Gs $\alpha$ peptides are immunogenic in patients with PMP and healthy donors. (A) T cell reactivity (proliferation) in blood samples from patients with PMP $(n=24)$. (B) T cell proliferation in healthy donors $(n=10)$. Peripheral blood mononuclear cells were prestimulated with mutated Gs $\alpha 30$-mer peptides (R201C and R201H). After 12-14 days, the T cells were restimulated with the mutated and WT Gs $\alpha$ peptides for 2 days before radioactive ${ }^{3} \mathrm{H}$-thymidine was added and the proliferation measured. A Stimulation Index of $\geq 2$ (above background) was considered positive. SEC3 superantigen was included as a positive control. Paired, two-tailed t-tests were used to calculate the statistical significance of T cell proliferation against WT Gs $\alpha$ peptide versus other conditions. (C) T cell reactivity (IFN- $\gamma$ production) in blood samples from patients with PMP ( $\mathrm{n}=7$ ). PBMCs were prestimulated with mutated Gs $\alpha 30$ mer peptides (R201C and R201H). After 12-14 days, the T cells were either tested directly (patients 559, 563, and 583) or previously prestimulated T cells were thawed (patients 549, 570, 576, and 581) and tested for IFN- $\gamma$ production in response to the mutated and WT Gs $\alpha$ peptides. SEC3 superantigen was included as a positive control. Statistically significant responses against Gs $\alpha$ peptide R201H compared with WT are indicated. Gs $\alpha$, guanine nucleotide-binding protein $\alpha$ subunit; IFN- $\gamma$, interferon gamma; PBMC, Peripheral blood mononuclear cell; PMP, pseudomyxoma peritonei; WT, wild type. 
A

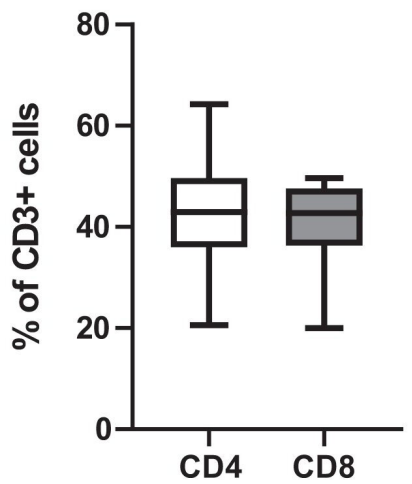

B

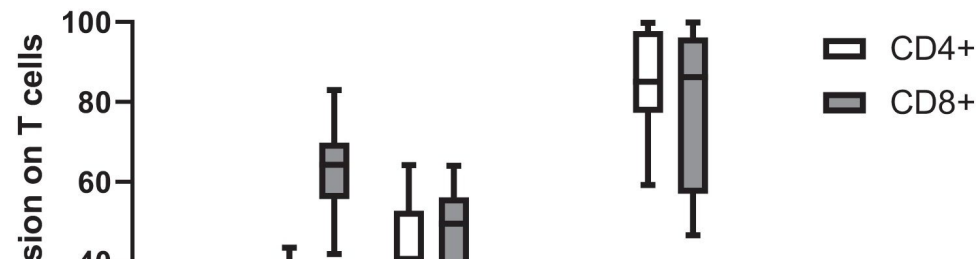

C

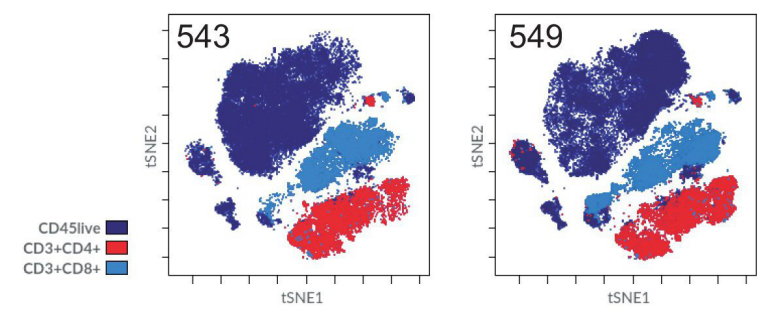

CD4

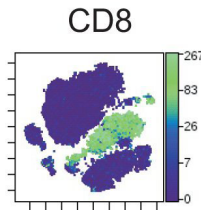

PD-1

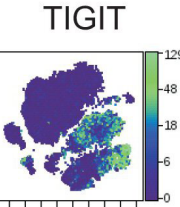

CXCR4

PMP 543
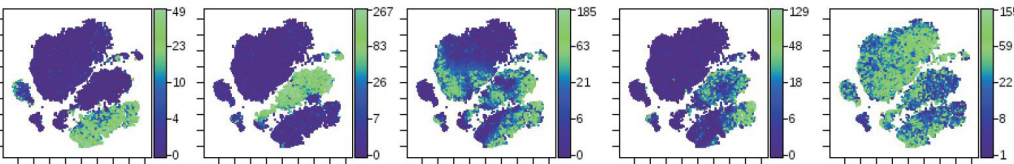

PMP 549
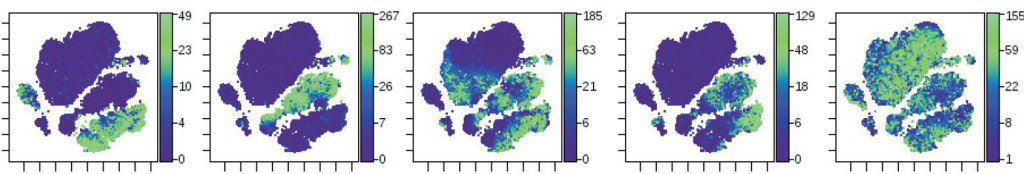

D

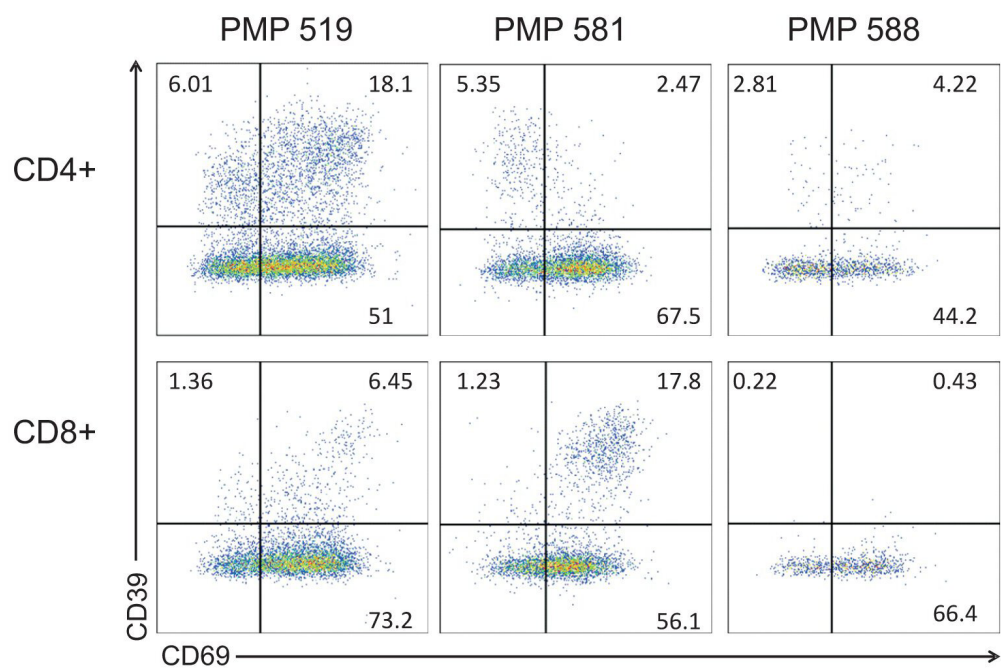

Figure 2 Immune checkpoint molecules/exhaustion markers are upregulated on tumor infiltrating T cells in patients with PMP $(n=18)$ analyzed by mass cytometry. (A) Percentage of CD4 + and CD8+ T cells (CD3+) in tumor samples from patients with PMP. The number of positive cells found in patient samples ranged between 766 and 69920 for CD3+, 284-14368 for CD4 + and 309-13960 CD8 + T cells. (B) Percentage of T cells expressing immune checkpoint molecules TIM-3, TIGIT, PD-1, LAG-3, and chemokine receptor CXCR4. (C) viSNE clustering analysis of PMP tumor from two representative patients showing CD4 + and CD8+ T-cell populations in combination with immune checkpoints PD-1 and TIGIT and chemokine receptor CXCR4. (D) Expression of activation/exhaustion markers CD39 and CD69 on T cells from PMP biopsies. Three patients (519, 581, and 588) had sufficient biopsy material for analysis of CD39 and CD69 expressions on T cells by flow cytometry. Top panels show the expression in CD4 + T cells and bottom panels show CD8 + T cell populations. LAG3, lymphocyte-activation gene 3; PD-1, programmed death receptor-1; PMP, pseudomyxoma peritonei; TIGIT, T cell immunoreceptor with Ig and ITIM. 
Pre-treatment

Insufficient immune response
Vaccination

Anti-tumor T cell expansion
Immune checkpoint inhibition

Restored immunity
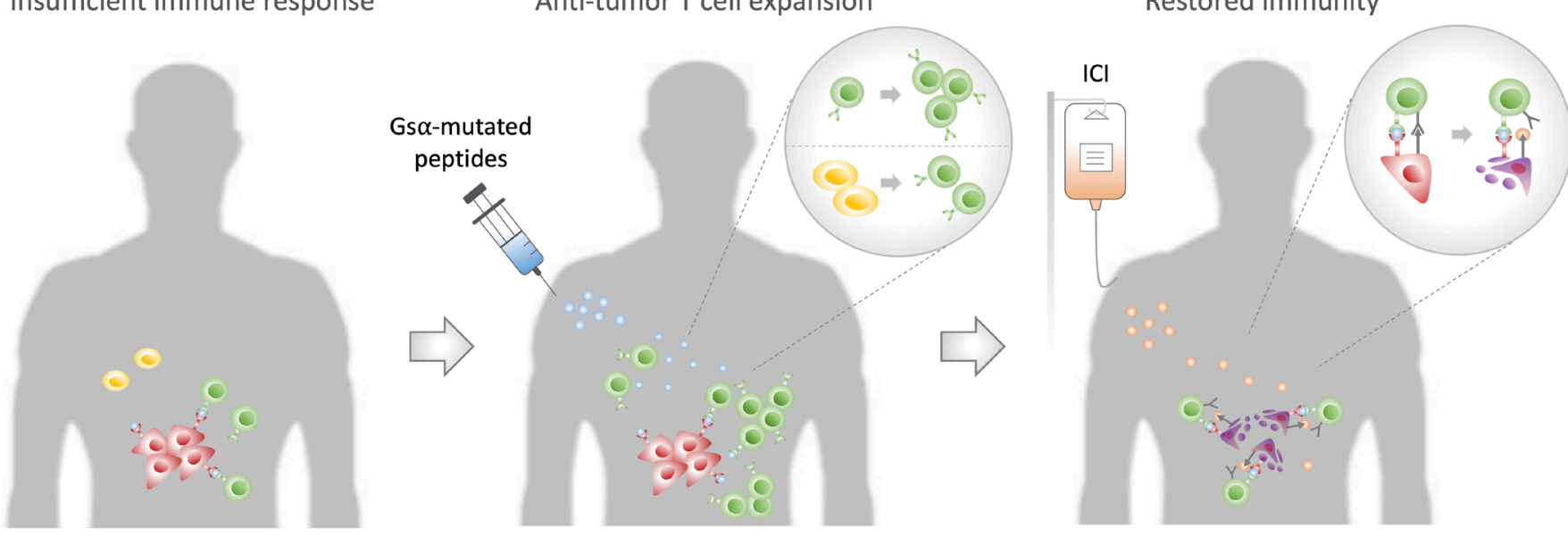

Naïve T cells

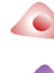

Antigen-experienced T cells

Tumor cell

Tumor cell death

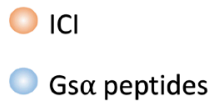

$\uparrow$ Tumor checkpoint protein

Y T cell checkpoint protein

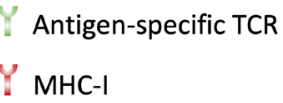

Antigen-specific TCR

Y MHC-I

Figure 3 Pseudovax hypothesis and therapeutic concept. Mutated Gs $\alpha$ peptides act as neoantigens and elicit a spontaneous, antitumor-specific T cell response in patients with PMP, but this is not sufficient to control tumor growth. We hypothesize that vaccination with mutated Gs $\alpha$ peptides will amplify the existing response and induce de novo responses of naïve $T$ cells, resulting in a clonal expansion of T cells recognizing mutated Gs $\alpha$. The antitumor immune response can then be boosted by adding an $\mathrm{ICl}$ to remove the inhibition caused by upregulation of immune checkpoint molecules on tumor-infiltrating $\mathrm{T}$ cells, restoring a functional immune response. Gs $\alpha$, guanine nucleotide-binding protein $\alpha$ subunit; ICl, immune checkpoint inhibitor; MHC-I, major histocompatibility complex class I; PMP, pseudomyxoma peritonei.

a preferential response against one of the peptides (proliferation value $>20 \%$ larger than towards the other peptide) was noted in 15 of the 18 responding cases; 9 and 6 samples favoring the R201C and R201H peptides, respectively. The WT peptide also elicited responses in the PMP samples in 8 of the 24 samples analyzed, but in all cases, the response to one or both mutant peptides was stronger. Concordance between the mutation detected in the tumor samples and a preferential response towards the corresponding peptide was observed in 7 of the 15 cases, where a preferential response was noted. For the healthy donors, responses were noted in 7 of the 10 analyzed samples (figure 1B and online supplemental table 2). The responses in healthy donors were generally of lower magnitude than in the patients with PMP. To further investigate the immunogenicity of the Gs $\alpha$ peptides, prestimulated $\mathrm{T}$ cells from seven patients with sufficient cell numbers were tested for IFN- $\gamma$ production in ELISPOT assays (figure $1 \mathrm{C}$ and online supplemental figure 1). These $\mathrm{T}$ cells were either thawed or freshly prestimulated $\mathrm{T}$ cells were used. The assay clearly showed higher responses for peptide R201H. Five of the patients harbored this specific mutation, whereas no mutation was detected for the last two (patients 559 and 583). The patients had previously shown somewhat higher or similar proliferative $\mathrm{T}$ cell responses to the R201C peptide.

\section{Analysis of T cell subsets in PMP samples}

Successful preparation of single cell suspensions from PMP samples with subsequent staining and analysis by CyTOF was achieved in 18/25 cases. The results clearly showed that most patients had CD3 + T cell infiltration (figure 2A). The CD4+:CD8 + T cell ratio was variable and in some cases did not make up $100 \%$ of the CD3 + population. Some of these CD3 + cells could be CD8-CD4NKT cells or double-negative T cells, also described to be immune suppressive. ${ }^{10}$ A large part of the infiltrating $\mathrm{T}$ cells seemed, however, to be antigen experienced as they expressed varying levels of immune checkpoint inhibitor molecules (figure 2B). The most predominantly expressed checkpoints were PD-1 and TIGIT domains, whereas TIM-3 (T cell immunoglobulin mucin-3) and LAG-3 were seen at very low levels in a few patients. PD-1 levels were similarly expressed by CD4 + and CD8 $+\mathrm{T}$ cells with an average expression of $40 \%-50 \%$. In contrast, TIGIT was predominantly expressed by CD8 $+\mathrm{T}$ cells (average of $60 \%$ ), whereas the average expression on CD4 + T cells was around $35 \%$, indicating that these $\mathrm{T}$ cells have seen their cognate antigen in vivo. ${ }^{11}$ viSNE clustering analysis from two representative patients with high levels of PD-1 and TIGIT expression is shown in figure 2C. The top plots show cells gated on live, CD45 + cells with clear CD8 + and CD4+ populations. The same clusters showed increased expression of PD-1 and TIGIT in particular; however, here in these patients CD4 + T cells exhibited the highest intensity of TIGIT staining. Some T cell populations expressed both TIGIT and PD-1. Interestingly, mass cytometry analysis showed a high percentage $(69 \%-$ 99\%) of CXCR4 (C-X-C chemokine receptor type 4) expression on infiltrating $\mathrm{T}$ cells, which may contribute to $\mathrm{T}$ cell homing to tumor. CXCR4 expression has been 
implicated in metastasis of several cancers, including in colorectal cancer, ${ }^{13}$ but is also important in lymphocyte trafficking. Its ligand-stromal cell-derived factor-1 (SDF1 and CXCL12) is produced in the tumor microenvironment. ${ }^{14}$ To further investigate $\mathrm{T}$ cells carrying these checkpoint molecules, we performed flow cytometry on biopsies from three patients with sufficient cell numbers available and stained $\mathrm{T}$ cells for expression of CD39 and CD69 (figure 2D). All patients displayed CD69+ $\mathrm{CD} 4+$ and CD8 $+\mathrm{T}$ cell populations and CD8 + T cells expressed less CD39+. The results were more variable with respect to $\mathrm{T}$ cell populations that were either CD39+ or CD39+CD69+, indicating that these markers may be interesting to investigate in a larger patient cohort.

\section{DISCUSSION}

The proliferative response that was observed when T cells from patients with PMP were stimulated with mutated Gs $\alpha$ peptides was exceptionally strong, suggesting the presence of a pre-existing immune response against mutated Gs $\alpha$. The mutated Gs $\alpha$ peptide inducing the strongest IFN- $\gamma$ response did not always correlate with the preferred peptide for the proliferative response but was consistently stronger for the mutated peptides compared with the WT peptide. However, proliferative responses and IFN- $\gamma$ ELISPOT responses could not be consistently compared as very few patients could be tested for IFN- $\gamma$ production. In healthy donors, the proliferative responses were much less pronounced but detectable in some cases. As $\mathrm{T}$ cells from healthy donors would not be expected to have been previously primed against mutated protein in vivo, the detection of Gs $\alpha$-specific $\mathrm{T}$ cell responses in these samples demonstrates the strong immunogenicity of the peptides. In some cases, the WT peptide also elicited proliferative responses in patients with PMP samples, although of lesser magnitude than the mutated peptides. Similar observations have been made in other vaccine trials where the peptides in question carried point mutations only. For instance, in a cancer trial of pancreatic patients vaccinated with a mix of mutated KRAS peptides, a persisting cross-reactivity ( $>10$ years postsurgery and vaccination) against the WT peptide, which was not included in the vaccine, was seen in two patients. ${ }^{15}$ No autoimmune adverse effects were observed, and it was speculated that this effect could actually have contributed to the strong memory $\mathrm{T}$ cell responses that were observed against the vaccine. Other studies on mutated KRAS epitopes have also confirmed in vitro reactivity against WT KRAS, in addition to the mutated epitope by $\mathrm{T}$ cell clones or TCR-modified $\mathrm{T}$ cells, and this could indeed be similar for Gs $\alpha$ peptides carrying point mutations. ${ }^{16-18}$ Our findings suggest that mutated Gs $\alpha$ acts as a shared (public) tumor neoantigen, resulting in immune responses that are readily detectable in patients with PMP. Because the described GNAS mutations are extremely frequent in patients with PMP, a vaccine would not have to be individually adapted for each patient as is the case for private neoantigens. Also, a strategy directed at Gs $\alpha$ would be tumor specific, since GNAS is mutated only in cancer cells, and no immune tolerance would be expected to develop, as is often seen for 'self-antigens' overexpressed in the tumor. Moreover, since GNAS is considered to be a driver oncogene, its expression is not likely to be lost by the tumor cells. ${ }^{19}$ Not only is GNAS a potentially ideal vaccination target, but also PMP seems to be an ideal disease for a vaccination strategy. Because PMP is a slow-growing cancer, vaccine treatment could be administered in the recurrent setting but also as adjuvant treatment before or after CRS-HIPEC. The adjuvant setting has previously been shown to be an appropriate situation for a therapeutic cancer vaccine strategy, where vaccination could consolidate the effect of surgery and prevent disease relapse. ${ }^{20}$ Taken together, these points demonstrate the high potential of Gs $\alpha$ as a candidate vaccine-targeted neoantigen in PMP. ${ }^{21}$

When the immune microenvironment of the same patients with PMP was interrogated, we found that for most of the PMP cases, infiltrating $\mathrm{T}$ cell populations could be detected and characterized. The only previous study of immune cells in PMP was performed by immunohistochemical staining of samples from 14 cases and reported varying infiltration of $\mathrm{T}$ cells $(\mathrm{CD} 3+)$, B cells $(\mathrm{CD} 20+)$, and macrophages (CD68+) in a low percentage of tumors. ${ }^{22}$ We chose to focus our analysis on the $\mathrm{T}$ cell subsets, as $\mathrm{T}$ cells can recognize specific neoantigens and would be the target cells for a peptide vaccine. Mass cytometry analysis showed that both CD4 + and CD8+ T cells infiltrated the PMP biopsies, and both T cell populations expressed PD-1 and TIGIT checkpoint receptors, suggesting that these $\mathrm{T}$ cells are antigen experienced and likely tumor reactive. Our preliminary results also suggest that additional markers of antigen-experienced $\mathrm{T}$ cells, such as CD39 and CD69, would be interesting to investigate further. The detection of strong $\mathrm{T}$ cell responses against mutated Gs $\alpha$ peptides (R201C or R201H) in the circulation of the majority of patients screened indicates that such antigen priming has indeed taken place in patients. PD-1 receptor engagement has an inhibitory effect on $\mathrm{T}$ cell effector functions, and high levels of PD-1 have been associated with $\mathrm{T}$ cell exhaustion and a dysfunctional phenotype. The inhibition can be caused by tumor intrinsic mechanisms or by cells or factors in the tumor microenvironment such as macrophages. ${ }^{23} 24$

Based on the current analyses of the immune cells in PMP and recent experience from previous vaccine trials, it is therefore likely that a Gs $\alpha$ peptide vaccine should be combined with immune checkpoint inhibition (ICI). Multiple preclinical studies have shown synergy between therapeutic vaccination and ICIs, and several ongoing clinical studies are currently evaluating this. ${ }^{25}$ Indeed, a recent study reported circulating CD8 $+\mathrm{T}$ cells double positive for PD-1 and TIGIT to be an early marker of therapeutic response to anti-PD-1 therapy in melanoma and Merkel cell carcinoma. ${ }^{26}$ From the perspective of currently known predictors of response to ICI, PMP is not 
an upfront ideal candidate for ICI monotreatment. In a total of 183 investigated cases in six individual studies, only one case was identified as MSI. ${ }^{27-32}$ Also, although a small number of cases have been extensively sequenced, there is no indication that PMP tumors have high tumor mutational burden. ${ }^{33}$ In this context, identification of the Gs $\alpha$ mutations as highly immunogenic points to an opportunity for patients with PMP to potentially benefit from immunotherapy in a combination treatment approach.

\section{CONCLUSION}

Taken together, our results suggest that Gs $\alpha$ peptides are highly immunogenic and may be used to reinforce a pre-existing immunity in patients with PMP and induce de novo immunity against mutated Gs $\alpha$. Analysis of the immune microenvironment of PMP tumor samples show that tumor infiltrating $\mathrm{T}$ cells are likely to have been antigen exposed, based on the expression of immune checkpoint molecules. These results form the rationale for the planned Pseudovax trial (protocol in preparation), which will explore peptide vaccination with Gs $\alpha$ peptides in combination with ICI in a first-in-man, signalfinding clinical trial (trial concept outlined in figure 3). In the longer term this treatment strategy may provide a possibility to offer a curative treatment to patients with PMP and for patients suffering from other GNAS-mutated cancers.

Acknowledgements We thank Solveig Mjelstad Olafsrud and Birthe Mikkelsen Saberniak for excellent technical assistance with pseudomyxoma peritonei (PMP) biopsies and T-cell assays, and Tove Øyjord, Martina Landschoof Skrede, Ina Katrine Nitschke Pettersen, and Arild Holth for excellent technical assistance in preparing PMP samples for molecular analyses. We also thank the Flow cytometry Core facility of OUS for providing technical assistance.

Contributors KF and EMl conceived, designed and supervised the study. KF and EMI wrote the manuscript. CLA, NM, and EMI created the figures. All coauthors contributed to respective parts of conducting experiments, data acquisition, analysis, and interpretation. All authors read and approved the contents of the manuscript. KF acts as guarantor.

Funding The study was funded by grants from the Norwegian Cancer Society (\#205716) and from Radium Hospital Foundation (\#182011) and Appendix Cancer Pseudomyxoma Peritonei Research Foundation (\#19010) to KF and South-Eastern Norway Regional Health Authority (\#2019004) to EMI.

Competing interests KF and EMI are inventors on a patent application filed EP21173961. All other authors declare no competing interests.

\section{Patient consent for publication Not applicable.}

Ethics approval The study was approved by the regional ethics committee of South-Eastern Norway (project ID \#10622 and \#30634), and written informed consent was required for participation.

Provenance and peer review Not commissioned; externally peer reviewed.

Data availability statement All data relevant to the study are included in the article or uploaded as supplementary information. Raw data can be made available upon request.

Supplemental material This content has been supplied by the author(s). It has not been vetted by BMJ Publishing Group Limited (BMJ) and may not have been peer-reviewed. Any opinions or recommendations discussed are solely those of the author(s) and are not endorsed by BMJ. BMJ disclaims all liability and responsibility arising from any reliance placed on the content. Where the content includes any translated material, BMJ does not warrant the accuracy and reliability of the translations (including but not limited to local regulations, clinical guidelines, terminology, drug names and drug dosages), and is not responsible for any error and/or omissions arising from translation and adaptation or otherwise.

Open access This is an open access article distributed in accordance with the Creative Commons Attribution Non Commercial (CC BY-NC 4.0) license, which permits others to distribute, remix, adapt, build upon this work non-commercially, and license their derivative works on different terms, provided the original work is properly cited, appropriate credit is given, any changes made indicated, and the use is non-commercial. See http://creativecommons.org/licenses/by-nc/4.0/.

\section{ORCID iDs}

Kjersti Flatmark http://orcid.org/0000-0001-7409-0780

Annette Torgunrud http://orcid.org/0000-0001-5751-9549

Karianne G Fleten http://orcid.org/0000-0003-0697-9750

Ben Davidson http://orcid.org/0000-0003-3332-8427

Nadia Mensali http://orcid.org/0000-0002-3130-4352

Christin Lund-Andersen http://orcid.org/0000-0002-7277-6612

Else Marit Inderberg http://orcid.org/0000-0002-6147-3536

\section{REFERENCES}

1 Chua TC, Moran BJ, Sugarbaker PH, et al. Early- and long-term outcome data of patients with pseudomyxoma peritonei from appendiceal origin treated by a strategy of cytoreductive surgery and hyperthermic intraperitoneal chemotherapy. J Clin Oncol 2012;30:2449-56.

2 Sørensen O, Flatmark K, Reed W, et al. Evaluation of complete cytoreductive surgery and two intraperitoneal chemotherapy techniques in pseudomyxoma peritonei. Eur J Surg Oncol 2012;38:969-76.

3 Kusamura S, Barretta F, Yonemura Y, et al. The role of hyperthermic intraperitoneal chemotherapy in pseudomyxoma peritonei after cytoreductive surgery. JAMA Surg 2021;156:e206363.

4 Lund-Andersen C, Torgunrud A, Fleten KG. Omics analyses in peritoneal metastasis - utility in the management of peritoneal metastases from colorectal cancer and pseudomyxoma peritonei: a narrative review. J Gastroint Oncol 2020.

5 Innamorati G, Wilkie TM, Kantheti HS, et al. The curious case of Gos gain-of-function in neoplasia. BMC Cancer 2018;18:293.

6 O'Hayre M, Vázquez-Prado J, Kufareva I, et al. The emerging mutational landscape of $\mathrm{G}$ proteins and $\mathrm{G}$-protein-coupled receptors in cancer. Nat Rev Cancer 2013;13:412-24.

7 Sugarbaker PH. Epithelial appendiceal neoplasms. Cancer J 2009;15:225-35.

8 Carr NJ, Cecil TD, Mohamed F, et al. A consensus for classification and pathologic reporting of pseudomyxoma peritonei and associated appendiceal neoplasia: the results of the peritoneal surface Oncology Group International (PSOGI) modified Delphi process. Am J Surg Pathol 2016;40:14-26.

9 Inderberg-Suso E-M, Trachsel S, Lislerud K, et al. Widespread CD4+ T-cell reactivity to novel hTERT epitopes following vaccination of cancer patients with a single hTERT peptide GV1001. Oncoimmunology 2012;1:670-86.

10 Prins RM, Incardona F, Lau R, et al. Characterization of defective CD4-CD8- T cells in murine tumors generated independent of antigen specificity. J Immunol 2004;172:1602-11.

11 Simon S, Labarriere N. PD-1 expression on tumor-specific T cells: friend or foe for immunotherapy? Oncoimmunology 2017;7:e1364828.

12 Simon S, Vignard V, Varey E, et al. Emergence of high-avidity melana-specific clonotypes as a reflection of anti-PD-1 clinical efficacy. Cancer Res 2017;77:7083-93.

13 Stanisavljević L, Aßmus J, Storli KE, et al. CXCR4, CXCL12 and the relative CXCL12-CXCR4 expression as prognostic factors in colon cancer. Tumour Biol 2016;37:7441-52.

14 Smit MJ, Schlecht-Louf G, Neves M, et al. The CXCL12/CXCR4/ ACKR3 axis in the tumor microenvironment: signaling, crosstalk, and therapeutic targeting. Annu Rev Pharmacol Toxicol 2021;61:541-63.

15 Wedén S, Klemp M, Gladhaug IP, et al. Long-term follow-up of patients with resected pancreatic cancer following vaccination against mutant K-ras. Int J Cancer 2011;128:1120-8.

16 Cafri G, Yossef R, Pasetto A, et al. Memory T cells targeting oncogenic mutations detected in peripheral blood of epithelial cancer patients. Nat Commun 2019;10:449.

17 Veatch JR, Jesernig BL, Kargl J, et al. Endogenous CD4+ $\mathrm{T}$ Cells Recognize Neoantigens in Lung Cancer Patients, Including Recurrent Oncogenic KRAS and ERBB2 (Her2) Driver Mutations. Cancer Immunol Res 2019;7:910-22. 
18 Yossef R, Tran E, Deniger DC, et al. Enhanced detection of neoantigen-reactive $T$ cells targeting unique and shared oncogenes for personalized cancer immunotherapy. JCl Insight 2018;3:1. doi:10.1172/jci.insight.122467

19 Bailey MH, Tokheim C, Porta-Pardo E, et al. Comprehensive characterization of cancer driver genes and mutations. Cell 2018;173:371-85.

20 van Willigen WW, Bloemendal M, Gerritsen WR, et al. Dendritic cell cancer therapy: vaccinating the right patient at the right time. Front Immunol 2018;9:2265.

21 Hollingsworth RE, Jansen K. Turning the corner on therapeutic cancer vaccines. NPJ Vaccines 2019;4:7.

22 Grizzi F, Cananzi FCM, Battista S. Immunohistochemical features of the pseudomyxoma peritonei microenvironment an opportunity for clinicians. Anal Quant Cytopatho 2018;40:109-15.

23 Chauvin J-M, Pagliano O, Fourcade J, et al. TIGIT and PD-1 impair tumor antigen-specific CD8 ${ }^{+} \mathrm{T}$ cells in melanoma patients. J Clin Invest 2015;125:2046-58.

24 Kwek SS, Lewis J, Zhang L, et al. Preexisting levels of CD4 T cells expressing PD-1 are related to overall survival in prostate cancer patients treated with ipilimumab. Cancer Immunol Res 2015;3:1008-16.

25 Hailemichael Y, Woods A, Fu T, et al. Cancer vaccine formulation dictates synergy with CTLA-4 and PD-L1 checkpoint blockade therapy. J Clin Invest 2018;128:1338-54.
26 Simon S, Voillet V, Vignard V, et al. PD-1 and TIGIT coexpression identifies a circulating CD8 $\mathrm{T}$ cell subset predictive of response to anti-PD-1 therapy. J Immunother Cancer 2020;8:e001631.

27 Borazanci E, Millis SZ, Kimbrough J, et al. Potential actionable targets in appendiceal cancer detected by immunohistochemistry, fluorescent in situ hybridization, and mutational analysis. $J$ Gastrointest Oncol 2017;8:164-72.

28 Gleeson EM, Feldman R, Mapow BL, et al. Appendix-derived pseudomyxoma peritonei (PMP): molecular profiling toward treatment of a rare malignancy. Am J Clin Oncol 2018;41:777-83.

29 Kabbani W, Houlihan PS, Luthra R, et al. Mucinous and nonmucinous appendiceal adenocarcinomas: different clinicopathological features but similar genetic alterations. Mod Pathol 2002;15:599-605.

30 Nummela P, Saarinen L, Thiel A, et al. Genomic profile of pseudomyxoma peritonei analyzed using next-generation sequencing and immunohistochemistry. Int $J$ Cancer 2015;136:E282-9.

31 Tokunaga R, Xiu J, Johnston C, et al. Molecular profiling of appendiceal adenocarcinoma and comparison with right-sided and left-sided colorectal cancer. Clin Cancer Res 2019;25:3096-103.

32 Zauber P, Berman E, Marotta S, et al. Ki-ras gene mutations are invariably present in low-grade mucinous tumors of the vermiform appendix. Scand J Gastroenterol 2011;46:869-74.

33 Pengelly RJ, Rowaiye B, Pickard K, et al. Analysis of mutation and loss of heterozygosity by whole-exome sequencing yields insights into pseudomyxoma peritonei. J Mol Diagn 2018;20:635-42. 